\title{
Faecal bile acid profiles in patients with large bowel cancer in Japan
}

\author{
Y HIKASA, N TANIDA, T OHNO, AND T SHIMOYAMA \\ From the Department of Internal Medicine 4, Hyogo College of Medicine, Nishinomiya, Hyogo, Japan
}

SUMmary To study the possible role of bile acids in carcinogenesis of the large bowel faecal bile acid profiles were analysed in detail in 14 patients with large bowel cancer and compared with 14 matched healthy subjects. The total faecal bile acid excretion was lower in the patients $(297.3 \pm 402.8 \mu \mathrm{mol} / \mathrm{day})$ than in the healthy subjects $(470.6 \pm 231.1 \mu \mathrm{mol} / \mathrm{day})(\mathrm{p}<0.01)$, although the difference became insignificant when these data were expressed as $\mu \mathrm{mol}$ per wet or dry faecal weight. The faecal profiles of individual bile acids and their conjugates were similar in both groups except that $5 \beta$-chol-3-en-24-oic acid, a bacterial metabolite of sulpholithocholic acid, was found in nine cancer patients $(64 \%)$ compared with only two healthy subjects $(14 \%)$ $(\mathrm{p}<0 \cdot 01)$. The significance of $5 \beta$-chol-3-en-24-oic acid in carcinogenesis needs further prospective and experimental studies.

Epidemiological studies suggest that the occurrence of large bowel cancer is closely related to dietary habits, especially to the consumption of meat or animal fat. ${ }^{12}$ In particular the effects of diet on faecal bile acids have been linked with carcinogenesis of the large bowel..$^{2-5}$ In clinical studies, faecal bile acid excretion has been reported to be increased in patients with large bowel cancer compared with healthy subjects, which provides support for the concept that bile acids may play a carcinogenic role. ${ }^{46}$ Several investigators, however, could not confirm this finding, ${ }^{7-10}$ one of the reasons for this discrepancy being the methodological problems involved in measuring faecal bile acids. Faecal bile acid profiles are complex because of bacterial deconjugation, dehydroxylation, oxidoreduction, and desulphation of bile acids in the intestine. ${ }^{11-13}$ In view of the suggested role of the intestinal microflora in pathogenesis of large bowel cancer, ${ }^{3-5}$ further studies of the microbial action on bile acids are needed to see whether any differences exist among populations with different risks of developing large bowel cancer, and also between healthy subjects and patients with cancer or precancerous conditions. Faecal bile acids in previous reports have been described in terms of the excretion of total or of the principal primary and

Address for correspondence: Dr N Tanida, Department of Internal Medicine 4, Hyogo College of Medicine, 1-1 Mukogawacho. Nishinomiya, Hyogo 663, Japan.

Received for publication 18 November 1983 secondary bile acids. ${ }^{4-10}$ Recently there has been considerable improvement in the methodology of faecal bile acid analysis, which enables more detailed profiles of individual bile acids and their mode of conjugation to be achieved. ${ }^{11}$ Therefore, it seems appropriate to re-analyse faecal bile acid profiles in patients with large bowel cancer or precancerous conditions. In our previous reports $^{12}{ }^{13}$ faecal bile acid profiles in the healthy subjects and patients with adenomatous polyps of the large bowel were studied and revealed that there was no significant difference between the two groups quantitatively or qualitatively. In this report we describe faecal bile acid profiles in patients with large bowel cancer in Japan and point out the possible significance of $5 \beta$-chol-3-en-24-oic acid, a bacterial metabolite of sulpholithocholic acid. ${ }^{14}$

\section{Methods}

\section{SUBJECTS}

Fourteen patients with large bowel cancer aged 45-74 years were studied. Age, sex, body weight, and faecal weights are summarised in Table 1 . The location of their tumours was as follows: caecum, one; ascending colon, one; transverse colon, two; sigmoid colon, seven; and rectum, three cases. The Dukes' classification ${ }^{15}$ was $\mathrm{A}$, six; B, three; and C, five cases respectively. The patients were referred to hospital for bloody stools, diarrhoea, or constipation. Their symptoms were mostly transient, and 
Table 1 Clinical data on patients with large bowel cancer and healthy subjects*

\begin{tabular}{lcc}
\hline & Patients & $\begin{array}{l}\text { Healthy } \\
\text { subjects }\end{array}$ \\
\hline Number of subjects & 14 & 14 \\
Age (yr) & $63 \cdot 4 \pm 8 \cdot 5$ & $63 \cdot 5 \pm 11 \cdot 8$ \\
Sex ratio (male/female) & $5 / 9$ & $5 / 9$ \\
Body weight $(\mathrm{kg})$ & $50 \cdot 6 \pm 7 \cdot 3$ & $51 \cdot 3 \pm 11 \cdot 7$ \\
Faecal wet weight (g/day) & $110 \cdot 1 \pm 140 \cdot 1 \dagger$ & $152 \cdot 8 \pm 79 \cdot 1 \dagger$ \\
Faecal dry weight (g/day) & $20 \cdot(0 \pm 20 \cdot 7 \dagger$ & $26 \cdot 1 \pm 11 \cdot 7 \dagger$ \\
\hline * The data are shown as mean \pm SD. & \\
† Significantly different between the two groups by median test \\
(p<0.05).
\end{tabular}

bloody stools were not observed during the faecal collection but faecal occult blood was present in three of Dukes A, one of Dukes B, and four of Dukes $C$ cases. At laparotomy all the tumours were proved to be adenocarcinoma. None of the patients in this study showed any signs of metastases clinically, by computed tomography scans, or at laparotomy. Patients who presented with stenosis or who had any previous history of gastroenterological disease were excluded from this study. Oral cholecystography before laparotomy revealed a functional gall bladder in all patients. Fourteen age, sex, and body weight matched volunteers without any past history of gastroenterological problems were studied for comparison (Table 1).

None of the subjects had received any drugs for at least two weeks before the faecal collection. Liver function tests and serum cholesterol levels were within normal limits. Diets of the subjects were not controlled but monitored by means of questionnaire retrospectively about their consumption or frequency of intake of cereals, meat, fish, vegetables, beverages, and tobacco as described before. ${ }^{16}$ Consent was obtained from all subjects, who were fully informed of the aims of the study.

CHEMICALS AND REFERENCE COMPOUNDS

The sources and purity of the chemicals, solvents, and reference bile acids were reported previously. $^{12} 17$ In addition lithocholic acid was purchased from Aldrich Chemical (Milwaukee, WIS, USA) for synthetic work.

$5 \beta$-Chol-3-en-24-oic acid was synthesised according to the modified method of Fieser and Ettorre as described by Issidorides et al. ${ }^{18}$ Thus, $8 \cdot 6$ mmol lithocholic acid was methylated with diazomethane and further processed as described, yielding $1.2 \mathrm{~g}$ of the product. This product gave a single spot on thin layer chromatography and three peaks on $12.5 \mathrm{~m}$ SE-30 capillary column gas chromatography at $240^{\circ} \mathrm{C}$. The $\mathrm{Rf}$ value of the spot relative to lithocholic acid methyl ester was 1.86 on thin layer chromatography, using a 2,2,4trimethylpentane:ethyl acetate:acetic acid (10:10 $: 0 \cdot 1)$ solvent system. ${ }^{19}$ The retention times relative to $5 \alpha$-cholestane of the three peaks were $0 \cdot 84,1 \cdot 04$, and $1 \cdot 10$, and they constituted 1,68 , and $31 \%$ by proportion in their total areas on gas chromatography, respectively. Gas chromatography and mass spectrometry analysis revealed that the second peak was of $5 \beta$-chol-3-en-24-oic acid methyl ester (Fig. 1).

Sulpholithocolic acid was synthesised according to the method of Tserng and Klein. ${ }^{20}$

ANALYTICAL PROCEDURE

The faeces were collected for 72 hours, in the case of the patients it was during the first three days after admission. No particular restrictions were imposed on the healthy subjects whose faeces were collected at our laboratory. All faeces were stored at $-20^{\circ} \mathrm{C}$ immediately after voiding. The analytical procedure has been described previously, ${ }^{11}{ }^{12}$ and is outlined here briefly. Faecal homogenates were extracted by refluxing in organic solvents, then purified, and bile acids were separated to their mode of conjugation on Lipidex DEAP (Packard Instrument, Downers Grove, ILL, USA) into neutral, unconjugated, glycine conjugated, taurine conjugated, and sulphated fractions. Each fraction was treated appropriately and finally measured by capillary column gas chromatography and mass spectrometry. ${ }^{12}$ In some cases total ion current chromatography was used for quantifying $5 \beta$-chol-3-en-24oic acid based on the assumption that the total ion current response of 5 $\beta$-chol-3-en-24-oic acid methyl ester equalled that of lithocholic acid methyl ester. All analyses were performed in duplicate. The recovery rate of bile acids was quantitative and the variation between duplicates was $6 \cdot 9 \pm 3 \cdot 2 \%$. The results are shown as the means of duplicates without correcting for the recovery rate. The data were analysed with median test and $\chi^{2}$ test.

\section{Results}

Dietary monitoring did not indicate any significant difference between the two groups, details of which will be shown elsewhere. Faecal mass, both as wet and dry weight, was smaller in the patients than in the healthy subjects $(p<0 \cdot 05$; Table 1$)$.

The total daily excretion of faecal bile acids is shown in Figure 2. Although there was wide variation in the results, a significantly lower excretion of total bile acids was observed in the patients than in the controls $(p<0 \cdot 01)$. A similar trend was found when the data were expressed per 


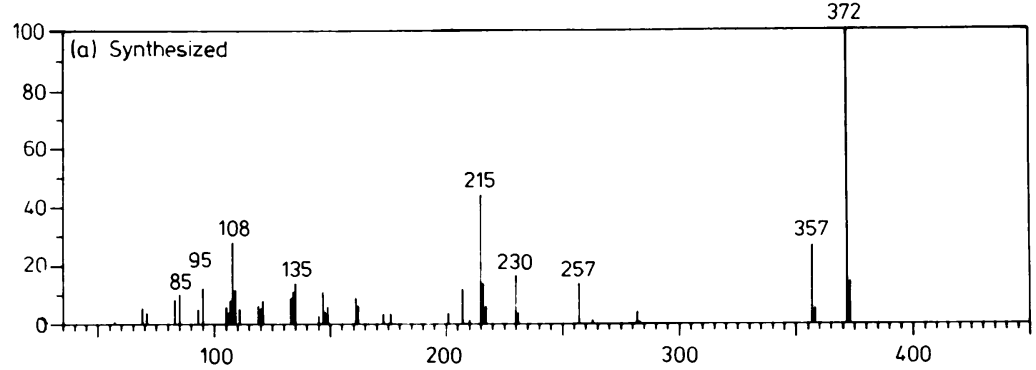

Fig. 1 Mass spectra of $5 \beta$ chol-3-en-24-oic acid methyl ester, $(A)$ synthesised according to modified method of Fieser and Ettorre as described by Issidorides et al, ${ }^{18}$ and $(B)$ found in faeces of patient with large bowel cancer. Their characteristics on gas chromatography and mass spectrometry were identical.

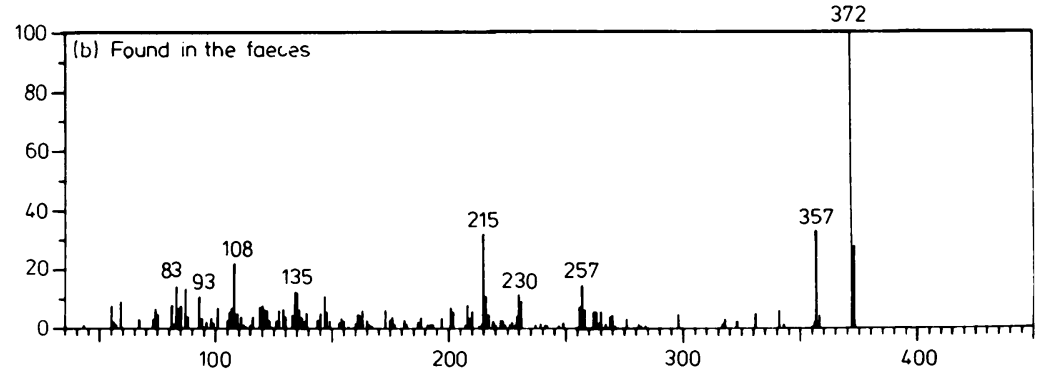

gram faecal wet and dry weight but the differences between the two groups became insignificant, reflecting the decreased output of faeces in the patients.

The mode of conjugation of the faecal bile acids in the two groups was also examined (Fig. 3). Nearly $90 \%$ of the total bile acids were in the unconjugated

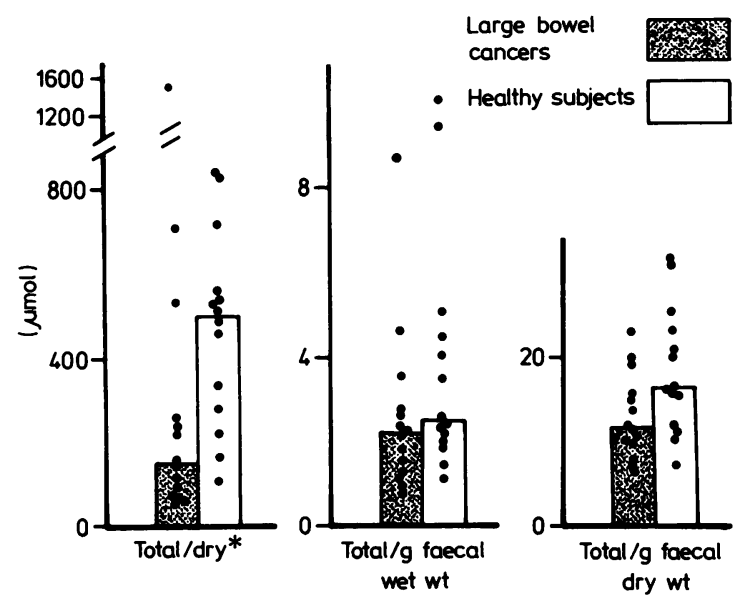

Fig. 2 Total faecal bile acid excretion in patients with large bowel cancer and healthy subjects. Each dot represents one subject and the columns indicate median values in each group. * Significantly different between the two groups with median test $(p<0 \cdot 01)$. fraction and there was no significant difference between the two groups.

When the composition of the individual bile acids in respect of their mode of conjugation was analysed as previously described, ${ }^{12}{ }^{13}$ the total excretion of lithocholic acid, chenodeoxycholic acid, cholic acid, and some secondary bile acids, but not deoxycholic

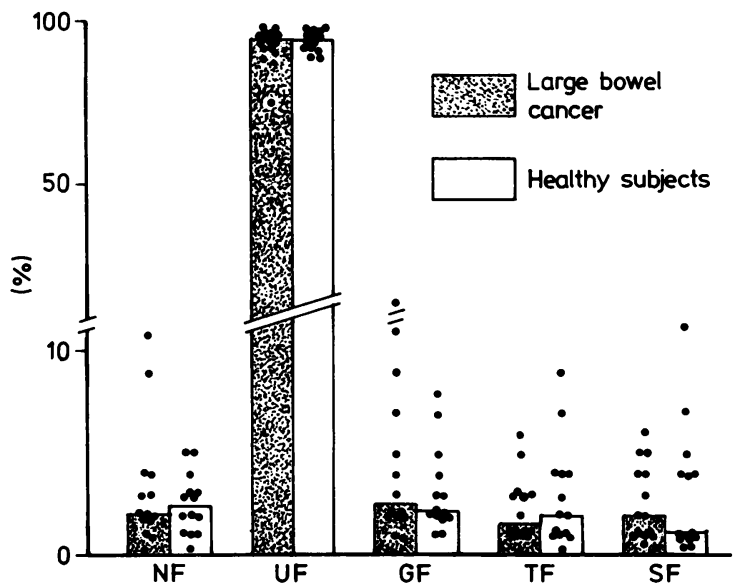

Fig. 3 Composition of faecal bile acids by their mode of conjugation in the patients with large bowel cancer and healthy subjects. Each dot represents one subject and columns indicate median values in each group. There was no significant difference between the two groups. NF: neutral fraction; UF: unconjugated fraction; GF: glycine conjugated fraction; TF: taurine conjugated fraction; $S F$ : sulphated fraction on Lipidex DEAP anion exchanger. 
acid, was lower in the patients than in the controls $(p<0.05)$. When the data were analysed per units of faecal weight, as with total faecal bile acid excretion, the differences became insignificant. There was no difference in the composition of the individual bile acids between the two groups except for the excretion of $5 \beta$-chol-3-en-24-oic acid in the unconjugated fraction, which was detected far more frequently and in higher amounts in the patients than in the controls $(p<0 \cdot 01$; Fig. 1 , Table 2). The excretion of $5 \beta$-chol-3-en-24-oic acid was not related to the age or sex of the patients, location and Dukes' classification of the tumours, nor to stool occult blood reaction.

Bile acid ratios, which were calculated from the original data to indicate the extents of dehydroxylation and oxidoreduction of bile acids - that is, formation of secondary bile acids - are summarised in Table 3. Considerable variation was observed but although the ratios tended to be higher in the patients than in the controls, the differences were not significant except for the oxidoreduction of chenodeoxycholic acid $(p<0.01)$. There was no association between the extent of oxidoreduction of chenodeoxycholic acid, total faecal bile acids, or $5 \beta$-chol-3-en-24-oic acid excretion.

\section{Discussion}

The incidence of large bowel cancer in Japan has been gradually increasing although it is still lower than that seen in western countries. ${ }^{21}$ Whether we are now experiencing the same trend as that in Japanese immigrants to the United States decades ago remains speculative. ${ }^{1}$ This unique situation makes it particularly important to study any aetiological factors which might be causing the apparent increase of large bowel cancer in Japan. The role of bile acids in carcinogenesis of the large bowel has been well discussed, ${ }^{2-6}$ and there is supporting evidence from experimental studies ${ }^{22-24}$ and clinical observations ${ }^{4-6}$ for the bile acid theory. The present study attempted to assess the carcino-

Table 2 Faecal excretion of 5 $\beta$-chol-3-en-24-oic acid in 14 patients with large bowel cancer and matched healthy subjects*

\begin{tabular}{lll}
\hline & Patients & Healthy subjects \\
\hline Frequency, casest & 9 & 2 \\
Range & trace $-11 \cdot 2$ & trace - trace \\
Median & 0.9 & trace \\
\hline
\end{tabular}

${ }^{*}$ Expressed as $\mu \mathrm{mol} / \mathrm{day}$, trace $=$ less than $0 \cdot 1 \mu \mathrm{mol} / \mathrm{day}$. + Significantly different between the two groups by $\chi^{2}$ test $(\mathrm{p}<0.01)$.
Table 3 Ratios of individual faecal bile acids indicating extent of dehydroxylation and oxidoreduction of faecal bile acids in 14 patients with large bowel cancer and matched healthy subjects*

\begin{tabular}{lcc}
\hline & Putients & $\begin{array}{l}\text { Healthy } \\
\text { subjects }\end{array}$ \\
\hline CDC + C/total & $0 \cdot 11 \pm(0 \cdot 18$ & $0 \cdot 15 \pm 1 \cdot 13$ \\
LC/CDC & $28 \cdot 6 \pm 36 \cdot 5$ & $5 \cdot 7 \pm 4 \cdot 5$ \\
DC/C & $2(0 \cdot 7 \pm 31 \cdot 7$ & $13 \cdot 5 \pm 17 \cdot 5$ \\
Epimers of LC/LC : & $(0 \cdot 2 \pm 0 \cdot 1$ & $0 \cdot 2 \pm() \cdot 1$ \\
Epimers of DC/DC & $0 \cdot 5 \pm 1 \cdot 3$ & $1 \cdot 6 \pm 4 \cdot 5$ \\
Epimers of CDC/CDC ${ }^{+}$ & $4 \cdot 6 \pm 2 \cdot 5$ & $3 \cdot 4 \pm 4 \cdot 2$ \\
Epimers of C/C & $3 \cdot 8 \pm 8 \cdot 7$ & $1 \cdot 6 \pm 2 \cdot()$ \\
\hline
\end{tabular}

* Expressed as mean $\pm \mathrm{SD}$ of the ratios calculated from total amounts of individual bile acids. CDC: chenodeoxycholic acid; C: cholic acid; LC: lithocholic acid; DC: deoxycholic acid. Epimers include the compounds having carbonyl group(s) in bile acid nucleus.

+ Significantly different between the two groups by median test $(\mathrm{p}<0 \cdot 01)$.

genic significance of bile acids by analysing faecal bile acid profiles in patients with large bowel cancer.

The total excretion of faecal bile acids in healthy subjects was comparable with our previous reports and with other studies dealing with Japanese subjects. $^{6} 121325$ Wide variation in the faecal bile acid excretion was evident in these studies, which was probably explained partly by the absence of faecal recovery markers and partly by ill-defined factors among which dietary variation was probably the most important. ${ }^{26}$ Some of these factors which caused variation in the faecal bile acid excretion might be also responsible for the decreased total excretion of faecal bile acids in cancer patients. Its precise mechanism could not be determined in the present study, however, differences in clinical situations between the two groups during the study were likely to have played a part. The lack of any increase in the excretion of total faecal bile acids in cancer patients observed in the present study is at variance with some previous reports ${ }^{4-6}$ but is similar to that reported in patients with large bowel cancer by several other groups. ${ }^{7-10}$ Our findings seem incompatible with the theory that large bowel carcinogenesis is based upon an increase in the total excretion of faecal bile acids.

Microbial metabolism of bile acids can be assessed indirectly by examining the degree of deconjugation, dehydroxylation, and oxidoreduction in faecal bile acids. The data indicated that these reactions were essentially the same in the patients with large bowel cancer as in the controls, except for reactions involving chenodeoxycholic acid. Although the exact mechanism is unknown, the degradation or catabolism of chenodeoxycholic acid 
by microorganisms appeared to be more extensive in the patients. It is interesting to note that chenodeoxycholic acid can be converted to lithocholic acid which in turn can be converted to $5 \beta$-chol-3-en-24oic acid by microbial action. ${ }^{14} 27$ As $5 \beta$-chol-3-en-24oic acid was found more frequently in the patient group than in the control group, further studies of the role of intestinal microflora in carcinogenesis of the large bowel should focus particularly on chenodeoxycholic acid and lithocholic acid metabolism.

The difference in 5 $\beta$-chol-3-en-24-oic acid excretion between the two groups is interesting. It has been shown that 5 $\beta$-chol-3-en-24-oic acid can be formed from sulpholithocholic acid during methylation with diazomethane. ${ }^{14}$ In the present study the sulphated bile acids were separated from unconjugated bile acids before the methylation. The stability of sulpholithocholic acid during the analytical procedure was further studied using the standard compound, which revealed no destruction of sulpholithocholic acid during the extraction and purification steps. Nor did processing of other major bile acids such as lithocholic acid, deoxycholic acid, chenodeoxycholic acid, and cholic acid produce any $5 \beta$-chol-3-en-24-oic acid. Therefore, artefactual formation was very unlikely.

Cholenoic acid has been reported in the faeces of patients with gall stones ${ }^{27}$ and was later identified as $5 \beta$-chol-3-en-24-oic acid formed from sulpholithocholic acid by microbial action. ${ }^{14}$ In the present study this compound was found exclusively in the unconjugated fractions of the faecal bile acids, which is in agreement with our previous observations. ${ }^{12}{ }^{13}$ Sulpholithocholic acid is secreted into bile as taurine or glycine conjugated form. ${ }^{28}$ Deconjugation of tauro-sulpholithocholic acid by intestinal microflora has been shown, ${ }^{29}$ and probably the liberated sulpholithocholic acid is used as a substrate for the bacterial enzyme producing $5 \beta$-chol-3-en-24oic acid. Some strains of Clostridia are known to be chiefly responsible for desulphation of sulpholithocholic acid. ${ }^{30}$ Several compounds have been reported as products of the desulphation of sulpholithocholic acid by microorganisms, including lithocholic acid, ${ }^{14} 3132$ its $3 \beta$-epimer, ${ }^{14}{ }^{30} 5 \beta$-cholanoic acid $^{30}$ and $5 \beta$-chol-3-en-24-oic acid ${ }^{14} 30$ together with other metabolites. ${ }^{14}$ As sulphation of bile acids has been considered to be one of the defence mechanisms against harmful compounds in the body, ${ }^{28}$ desulphation implies reconversion to possibly toxic compounds which might have pathological effects on the intestine.

Introduction of the double bond into the steroid nucleus during cholenoic acid formation is in accord with the hypothesis by Hill and his coworkers suggesting the production of carcinogens from bile acids by the intestinal microflora. ${ }^{34}$ Although $5 \beta$ chol-3-en-24-oic acid was detected in the faeces of healthy subjects, patients with large bowel cancer, and those with adenomatous polyps of the large bowel, ${ }^{12} 1.3$ we have been unable to detect any 3-oxo-nuclear dehydrogenated bile acids and it is not certain that $5 \beta$-chol-3-en-24-oic acid formation is compatible with the nuclear dehydrogenation described by Hill et al. ${ }^{4}$ The present study and our previous one suggest that $5 \beta$-chol-3-en-24-oic acid is associated with large bowel cancer but not with adenomatous polyps of the large bowel. ${ }^{13}$ Whether the formation of this compound is a cause or consequence of large bowel cancer remains to be determined. Experimental studies both in vitro and in vivo are needed to assess its possible significance in carcinogenesis of the large bowel.

Parts of this study were presented at the 24th Autumn and 69th Annual Meeting of Japanese Gastroenterological Society. This study was supported in part by a Grant-in-Aid for Cancer Research (55-1) from the Ministry of Health and Welfare of Japan. The authors wish to thank Dr G R Thompson, Professor R H Dowling, and Dr K D R Setchell for their critical comments and help in preparing this manuscript.

\section{References}

1 Haenszel W, Berg JW, Segi M, Kurihara M, Locke FB. Large-bowel cancer in Hawaiian Japanese. J Natl Cancer Inst 1973; 51: 1765-79.

2 Wynder EL, Reddy BS. Diet and cancer of the colon. Curr Concepts Nutr 1977; 6: 55-71.

3 Hill MJ, Crowther JS, Drasar BS, Hawksworth G, Aries V, Williams REO. Bacteria and aetiology of cancer of large bowel. Lancet 1971; 1: 95-100.

4 Hill MJ, Drasar BS, Williams REO et al. Faecal bile-acids and Clostridia in patients with cancer of the large bowel. Lancet 1975; 1: 535-9.

5 Wynder EL, Reddy BS. Metabolic epidemiology of colorectal cancer. Cancer 1974; 34: 801-6.

6 Reddy BS, Wynder EL. Metabolic epidemiology of colon cancer. Fecal bile acids and neutral sterols in colon cancer patients and patients with adenomatous polyps. Cancer 1977; 39: 2533-9.

7 Moskovitz M, White C, Barnett RN et al. Diet, faecal bile acids, and neutral sterols in carcinoma of the colon. Dig Dis Sci 1979; 24: 746-51.

8 Mudd DG, McKelvey STD, Norwood W, Elmore DT, Roy AD. Faecal bile acid concentrations of patients with carcinoma or increased risk of carcinoma in the large bowel. Gut 1980; 21: 587-90.

9 Murray WR, Blackwood A, Trotter JM, Calman KC, MacKay C. Faecal bile acids and Clostridia in the aetiology of colorectal cancer. Br J Cancer 1980; 41: 923-8. 
10 Perogambros A, Legakis NJ. Faecal bile acids in patients with colon cancer. Zentrabl Bakteriol [Orig B] 1982; 176: 346-8.

11 Setchell KDR, Lawson AM, Tanida N, Sjövall J. General methods for the analysis of metabolic profiles of bile acids and related compounds in feces. $J$ Lipid Res 1983; 24: 1085-1100.

12 Tanida N, Hikasa Y, Hosomi M, Satomi M, Ohama I, Shimoyama T. Fecal bile acid analysis in healthy Japanese subjects using a lipophilic anion exchanger, capillary column gas chromatography and mass spectrometry. Gastroenterol Jpn 1981; 16: 363-71.

13 Tanida N, Hikasa Y, Shimoyama T, Setchell KDR. Comparison of faecal bile acid profiles between patients with adenomatous polyps of the large bowel and healthy subjects in Japan. Gut 1984; 25: 824-32.

14 Kelsey MI, Molina JE, Huang SKS, Hwang KK. The identification of microbial metabolites of sulfolithocholic acid. J Lipid Res 1980; 21: 751-9.

15 Dukes CE. The classification of cancer of the rectum. $J$ Pathol Bact 1932; 35: 323-32.

16 Shimoyama T. Ohno T. Tanida N et al. Epidemiology and clinical features in colonic diverticulosis. Geriatr Med (Jpn) 1983; 21: 1213-23.

17 Tanida N, Hikasa Y, Shimoyama T. Evaluation of polyethylene glycol-HT as a stationary phase for capillary column gas-liquid chromatography of trimethylsilyl ethers of bile acid methyl esters. $J$ Chromatogr 1982; 240: 75-9.

18 Issidorides $\mathrm{CH}$, Fieser M, Fieser LF. Selenium dioxide oxidation of methyl $\Delta^{3}$-cholenate. J Am Chem Soc 1960; 82: 2002-5.

19 Eneroth P. Thin-layer chromatography of bile acids. $J$ Lipid Res 1963; 4: 11-6.

20 Tserng KY, Klein PD. Synthesis of sulfate esters of lithocholic acid, glycolithocholic acid, and taurolithocholic acid with sulfur trioxide-triethylamine. J Lipid Res 1977; 18: 491-5.

21 Lee JAH. Recent trends of large bowel cancer in Japan compared to United States and England and Wales. Int J Epidemiol 1976; 5: 187-94.
22 Narisawa T, Magadia NE, Weisburger JH, Wynder EL. Promoting effect of bile acids on colon carcinogenesis after intrarectal instillation of $N$-methyl- $N^{\prime}$ nitro- $N$-nitrosoguanidine in rats. $J$ Natl Cancer Inst 1974; 53: 1093-7.

23 Silverman SJ, Andrews AW. Bile acids: co-mutagenic activity in the Salmonella-mammalian-microsome mutagenicity test: Brief communication. J Natl Cancer Inst 1977; 59: 1557-9.

24 Takano S, Matsushima M, Ertürk E, Bryan GT. Early induction of rat colonic epithelial ornithine and $S$ adenosyl-L-methionine decarboxylase activities by $N$ methyl- $N$-nitro- $N$-nitrosoguanidine or bile salts. Cancer Res 1981; 41: 624-8.

25 Mower HF, Ray RM, Shoff R et al. Fecal bile acids in two Japanese populations with different colon cancer risks. Cancer Res 1979; 39: 328-31.

26 Cummings JH, Wiggins HS, Jenkins DJA et al. Influence of diets high and low in animal fat on bowel habit, gastrointestinal transit time, fecal microflora, bile acid, and fat excretion. J Clin Invest 1978; 61: 953-63.

27 Norman A, Palmer RH. Metabolites of lithocholic acid-24-C $\mathrm{C}^{14}$ in human bile and feces. J Lab Clin Med 1964; 63: 986-1001.

28 Palmer RH, Bolt MG. Bile acid sulfates. I. Synthesis of lithocholic acid sulfates and their identification in human bile. J Lipid Res 1971; 12: 671-9.

29 Bremmelgaard A, Bremmelgaard A. Bacterial metabolization of taurolithocholic acid 3- $\alpha$-sulfate. Acta Pathol Microbiol Scand [B] 1974; 82: 537-40.

30 Borriello SP, Owen RW. The metabolism of lithocholic acid and lithocholic acid-3- $\alpha$-sulfate by human fecal bacteria. Lipids 1982; 17: 477-82.

31 Imperato TJ, Wong CG, Chen LJ, Bolt RJ. Hydrolysis of lithocholate sulfate by Pseudomonas aeruginosa. $J$ Bacteriol 1977; 130: 545-7.

32 Huijghebaert SM, Mertens JA, Eyssen HJ. Isolation of a bile salt sulfatase-producing Clostridium strain from rat intestinal microflora. Appl Environ Microbiol 1982; 43: $185-92$. 